\title{
Temperature Dependence of Ultra-Exothermic Charge Recombinations**
}

\author{
Carlos Serpa,* Paulo J. S. Gomes, Luis G. Arnaut,* J. Seixas de Melo, and \\ Sebastião J. Formosinho ${ }^{[a]}$
}

We measured the temperature dependence (from +32 to $-50^{\circ} \mathrm{C}$ ) of charge-recombination rates between contact radical ion pairs in isopropyl ether. In the systems selected for this study, aromatic hydrocarbon cations are the electron acceptors and the fumaronitrile anion is the electron donor. Nearly quantitative electron transfers occur at all temperatures. The charge recombinations have excess exothermicities of $-60 \mathrm{kcal} \mathrm{mol}^{-1}$ and exhibit a very weak temperature dependence. Our observations emphasize the absence of solvent effects and the relevance of nuclear tunneling in charge recombinations.

\section{Introduction}

The temperature dependence of chemical reactions provides important insights into the molecular factors that influence their rates. Experimental studies on the temperature dependence of electron-transfer reactions have followed the leads given by classical theory on the change from 1) activated reactions at small exothermicities (the "normal region") to 2) activationless reactions at moderate exothermicities and, again, to 3) activated reactions at high exothermicities (the Marcus "inverted region"). ${ }^{[1]}$ The work by Liang et al. was a landmark in these experimental studies because the rates of intramolecular charge shifts-from a biphenylyl group to a covalently linked naphthyl group from quinone-were studied from 100 to $-94^{\circ} \mathrm{C}^{[2,3]}$ The activated regime was observed for weakly exothermic charge shifts, but the rates in the inverted region were virtually independent of the temperature. Verhoeven and coworkers obtained similar results for intramolecular charge recombinations following photoinduced charge separation in rigidly bridged donor-acceptor molecules. ${ }^{[4]}$ This work is especially pertinent to the present study because it also employed naphthalene derivatives and nitriles as the redox active species. In particular, the lifetime of the charge-separated state of their bridged system, where the dimethoxynaphthalene cation is separated by four sigma bonds $\left(4.6 \AA^{[5]}\right)$ from the dicyanoethylene anion, remained constant at $8 \mathrm{~ns}$, even when the temperature was lowered to $-23^{\circ} \mathrm{C}$. Another influential study was carried out by Miyasaka et al. ${ }^{[6]}$ who showed that charge recombinations in porous glass follow the same free-energy relationships as those observed in polar solvents and that these relationships are maintained at $-196^{\circ} \mathrm{C}(77 \mathrm{~K})$. These authors found a weak temperature dependence of the rates from room temperature down to about $-130^{\circ} \mathrm{C}$ and assigned it to the decrease or freezing of the low-frequency modes, in particular, to the intermolecular vibration of the two radical ions. The temperature dependence of the nonradiative decay of intramolecular exciplexes with aromatic hydrocarbons as accept- ors and amines as donors ${ }^{[7,8]}$ has also been studied in detail and small activation energies were found for such systems.

The activationless regime of the electron transfers in the inverted region cannot be explained by the classical Marcus theory, ${ }^{[9]}$ which predicts a rate decrease-of several orders of magnitude-as the temperature is lowered. It can, however, be described by the golden rule of quantum mechanics, ${ }^{[10-13]}$ which formulates the rates as the product of a temperature-independent electronic factor $(V)$ and a temperature-dependent Franck-Condon factor $(J)$ [Eqs. (1) and (2)]:

$k=\frac{2 \pi}{h}|V|^{2} J$

$J=\frac{1}{\sqrt{4 \pi \lambda_{\mathrm{s}} k_{\mathrm{B}} T}} \sum_{n=0}^{\infty} e^{-s} \frac{S^{n}}{n !} \exp \left[-\frac{\left(\Delta G^{0}+\lambda_{\mathrm{s}}+n h v_{\mathrm{v}}\right)^{2}}{4 \lambda_{\mathrm{s}} k_{\mathrm{B}} T}\right]$

where the donor and acceptor are represented by a single high frequency $\left(h v_{\mathrm{v}}=h v_{\mathrm{D}}=h v_{\mathrm{A}}=1500 \mathrm{~cm}^{-1}\right)$, the reorganization energy of the high-frequency vibrational modes is represented by $\lambda_{\mathrm{v}}$, and $S=\lambda_{\mathrm{v}} /\left(h v_{\mathrm{v}}\right)$. Except for very high temperatures, these parameters are not appreciably dependent on the temperature. However, the reorganization energy of the solvent, which can be estimated using the dielectric continuum

[a] Dr. C. Serpa, P. J. S. Gomes, Prof. L. G. Arnaut, Dr. J. S. de Melo, Prof. S. J. Formosinho

Department of Chemistry, University of Coimbra 3000-535 Coimbra (Portugal)

Fax: (+ 35) 1-239-827703

E-mail: serpasoa@ci.uc.pt lgarnaut@ci.uc.pt

[**] Dedicated to Professor Richard A. Caldwell, on the occasion of his retirement, with the expression of our appreciation for his contributions to photochemistry.

Supporting information for this article is available on the WWW under http://www.chemphyschem.org or from the author. 
approximation [Eq. (3)]:

$\lambda_{\mathrm{s}}=e^{2}\left(\frac{1}{2 r_{+}}+\frac{1}{2 r_{-}}-\frac{1}{r}\right)\left(\frac{1}{n_{\mathrm{D}}^{2}}-\frac{1}{\varepsilon}\right)$

where $r_{+}$and $r_{-}$are the effective radii of the two radical ions, and $r$ is the distance between their centers, depends on the temperature because the refractive index $\left(n_{\mathrm{D}}\right)$ and the dielectric constant $(\varepsilon)$ of the solvent increase differently as the temperature is lowered. The golden rule can only reproduce the temperature independence of the rates in the inverted region if the reorganization energy is independent of the temperature. This would suffice to elucidate which molecular factors influence the electron-transfer rates if $\Delta G^{0}$ would be independent of the temperature. However, the Gibbs energy for charge recombination, $\Delta G_{C R}^{0}$, also changes with the temperature [Eq. (4)]: $:^{[14]}$

$\Delta G_{\mathrm{CR}}^{0}=-\left[E_{\mathrm{D}}^{\mathrm{ox}}-E_{\mathrm{A}}^{\mathrm{red}}-\frac{\mu^{2}}{\rho^{3}}\left(\frac{\varepsilon-1}{2 \varepsilon+1}-\frac{n_{\mathrm{D}}-1}{2 n_{\mathrm{D}}+1}\right)+0.38\right]$

where $\Delta G^{0}$ is expressed in $\mathrm{eV}, E_{\mathrm{D}}{ }^{\text {ox }}$ and $E_{\mathrm{A}}{ }^{\text {red }}$ are the redox potentials of the donor and the acceptor, and $\mu$ and $\rho$ are the exciplex dipolar moment and the solvent cavity radius, respectively $\left(\mu^{2} / \rho^{3}\right.$ has a typical value of $\left.0.75 \mathrm{eV}\right)$. The free-energy dependence of the rates and the temperature dependence of $\Delta G^{0}$ may fortuitously compensate for the temperature dependence of $\lambda_{s}$. Additionally, the temperature dependence of the redox potential is usually not known.

We selected for this study the charge recombinations between the radical cation of pyrene or naphthalene and the radical anion of fumaronitrile in $n$-heptane and isopropyl ether, because the free energies of these charge recombinations range between -60 and $-80 \mathrm{kcal} \mathrm{mol}^{-1}$ and occur at a region where the rates are very weakly dependent on the driving forces. ${ }^{[15]}$ Moreover, as the separation of these ions is endoenergetic, in $n$-heptane and isopropyl ether they exist as nearly pure contact radical ion pairs (CRIP) with a very weak (exciplex) emission, and their decays are dominated by the charge recombination. $^{[15]}$ These systems give us the opportunity to focus on the temperature dependence of the reorganization energies because the free-energy dependence of the rates is negligible within $\pm 5 \mathrm{kcal} \mathrm{mol}^{-1}$.

\section{Results}

The photoinduced charge separation, from electronically excited aromatic hydrocarbons (pyrene, Py; naphthalene, $\mathrm{Na}$ ) to fumaronitrile (FN), and subsequent charge recombination in the exciplex formed in weakly polar solvents and supercritical $\mathrm{CO}_{2}$ follows the mechanism illustrated in Scheme 1. We have shown in detail that the exciplexes formed between these species have nearly quantitative charge separations ${ }^{[15]}$ and that in $n$-heptane, the fluorescence and triplet-state formation are minor decay channels. The fluorescence quantum yields are 0.102 and 0.0044 for the Py/FN and $\mathrm{Na} / \mathrm{FN}$ radical ion pairs, respectively, and the corresponding triplet quantum yields are 0.06 for Py/FN and $<0.01$ for $\mathrm{Na} / \mathrm{FN}^{\left[{ }^{[15]}\right.}$ The energy balance ob-

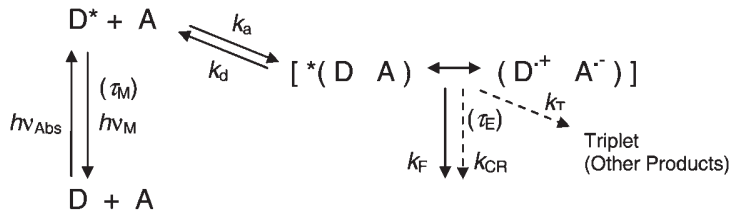

Scheme 1. CRIP formation and decay with the indication of the kinetic rates and lifetimes associated with the different processes. $\tau_{\mathrm{M}}=$ monomer intrinsic lifetime, $\tau_{\mathrm{E}}=$ CRIP decay lifetime, $k_{\mathrm{a}}=\mathrm{CRIP}$ "association" rate; $k_{\mathrm{d}}=\mathrm{CRIP}$ "dissociation" rate; $k_{\mathrm{F}}=\mathrm{CRIP}$ florescence rate, $k_{\mathrm{CR}}=$ charge recombination rate, $k_{\mathrm{T}}=$ intersystem crossing rate.

tained in photoacoustic calorimetry measurements reveal that the quantum yields for the formation of other products must be less than $0.01 .^{[15]}$ Consequently, the charge recombination quantum yields within the $\mathrm{Py} / \mathrm{FN}$ and $\mathrm{Na} / \mathrm{FN}$ radical ion pairs are 0.84 and 0.996 , respectively. Although these measurements refer to $n$-heptane, Van Haver et al. have shown that the intersystem crossing quantum yields decrease with an increase in solvent polarity, ${ }_{1}^{[16]}$ which reassures us that the charge recombination quantum yields cited above are lower limits for the systems addressed herein.

The CRIP formation by photoinduced electron transfer follows, very closely, the simplified Smoluchowski equation for diffusion-controlled reactions (see Supporting Information). The temperature dependence of the photoinduced charge separation gives activation energies that cannot be distinguished from those of diffusion. With dynamic studies in isopropyl ether we obtain $2.9 \mathrm{kcalmol}^{-1}$ for Py/FN and $2.3 \mathrm{kcal} \mathrm{mol}^{-1}$ for $\mathrm{Na} / \mathrm{FN}$.

The separation between monomer and exciplex emissions is larger in isopropyl ether than in $n$-heptane, as shown in Figure 1 for the exciplex formed between naphthalene and fumaronitrile. The degree of charge separation can be related to the separation between monomer and exciplex emissions. ${ }^{[17]}$ For the exothermic exciplex formation, a nearly full-charge separation is expected. ${ }^{[15]}$ For the exciplex formed by pyrene and fumaronitrile, the emission maximum is displaced from $440 \mathrm{~nm}$ in $n$-heptane to $505 \mathrm{~nm}$ in isopropyl ether, also reducing the overlap with the monomer emission. Figure 1 illustrates the sensitivity of the exciplex emission to solvents of different po-

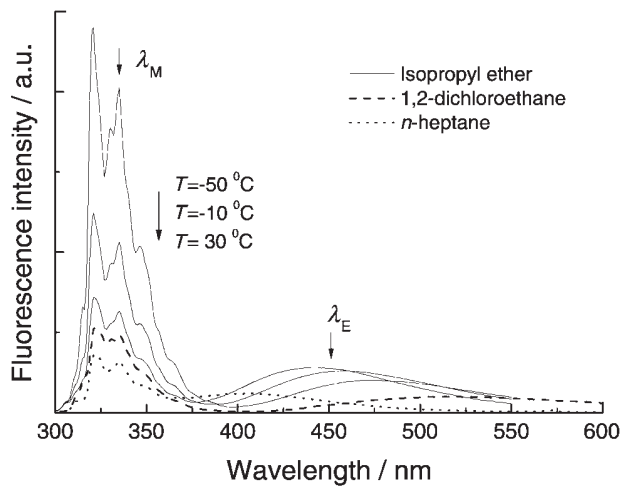

Figure 1. Fluorescence of naphthalene in the presence of fumaronitrile in $n$ heptane $(\cdots . .$.$) , 1,2-dichloroethane (-----), and isopropyl ether at different$ temperatures (-). 
larity and to isopropyl ether at different temperatures. Temperature changes the dielectric properties of isopropyl ether from $\varepsilon=3.639$ and $n_{\mathrm{D}}=1.361$ at $30^{\circ} \mathrm{C}$ to $\varepsilon=8.656$ and $n_{\mathrm{D}}=1.418$ at $-50^{\circ} \mathrm{C}{ }^{[18]}$ The observed exciplex emission shift is characteristic of charge-separated species with a high degree of charge transfer. ${ }^{[19]}$ The separation of the radical ion pair remains thermodynamically unfavorable in isopropyl ether and the mechanism demonstrated for the reactions in $n$-heptane and supercritical $\mathrm{CO}_{2}$ can then be applied to the interpretation of monomer and exciplex decays in isopropyl ether. Additionally, the fluorescence quantum yield of the exciplexes in isopropyl ether is only about 0.04 for Py/FN and 0.016 for $\mathrm{Na} / \mathrm{FN}$. These values are of the same magnitude-or even smaller-than those found in $n$-heptane and provide further evidence of the nearly quantitative charge transfer in the exciplexes. This charge-separated state can then be characterized as a CRIP in isopropyl ether. The weak fluorescence of the charge-separated species permits the measurement of charge recombination rates and results from a subtle balance between large extents of charge transfer in weakly polar solvents and a measurable emission.

To investigate the kinetics of charge recombination within the CRIP, we used time-correlated single-photon counting (TCSPC). Time-dependent intensity profiles were collected in the monomer, $I_{M}(t)$, and exciplex, $I_{E}(t)$, emission regions. The emission wavelengths are indicated in Figure 1 for the Na/FN CRIP. The mechanism presented in Scheme 1 can be resolved with the method described by Birks. ${ }^{[2]}$ The time-dependent intensity profiles are given by Equations (5) and (6):

$I_{\mathrm{M}}(t)=a_{11} e^{-\lambda_{1}}+a_{12} e^{-\lambda_{2}}$

$I_{\mathrm{E}}(t)=a_{21} e^{-\lambda_{1}}+a_{22} e^{-\lambda_{2}}$

where $\lambda_{1}$ and $\lambda_{2}$ are the reciprocal of the decay times $\tau_{1}$ and $\tau_{2}$ and are given by Equation (7):

$2 \lambda_{2,1}=\left(k_{X}+k_{Y}\right) \pm \sqrt{\left(k_{X}+k_{Y}\right)^{2}+4 k_{a} k_{d}}$

with $k_{X}=k_{a}+1 / \tau_{\mathrm{M}}$ and $k_{Y}=k_{d}+1 / \tau_{\mathrm{E}}$. The experimental data obtained from the decays given by Equations (5) and (6) $\left(\tau_{1}, \tau_{2}\right.$, and the ratio of the pre-exponential factors $a_{12} / a_{11}$ at the monomer region), together with the value of $\tau_{\mathrm{M}}$, whose temperature dependence was obtained independently by measurements in a dilute solution of pyrene or naphthalene in the absence of fumaronitrile, allow the determination of all rate constants in Scheme 1 through Equations (8)-(10). Representative decays are shown in Figure 2.

$$
\begin{aligned}
& k_{a}=\frac{\lambda_{1}+\left(\frac{a_{12}}{a_{11}}\right) \lambda_{2}}{\left(\frac{a_{12}}{a_{11}}\right)+1}-k_{M} \\
& k_{d}=\frac{k_{X} k_{Y}-\lambda_{1} \lambda_{2}}{k_{a}} \\
& k_{E}=k_{d}-k_{Y}
\end{aligned}
$$

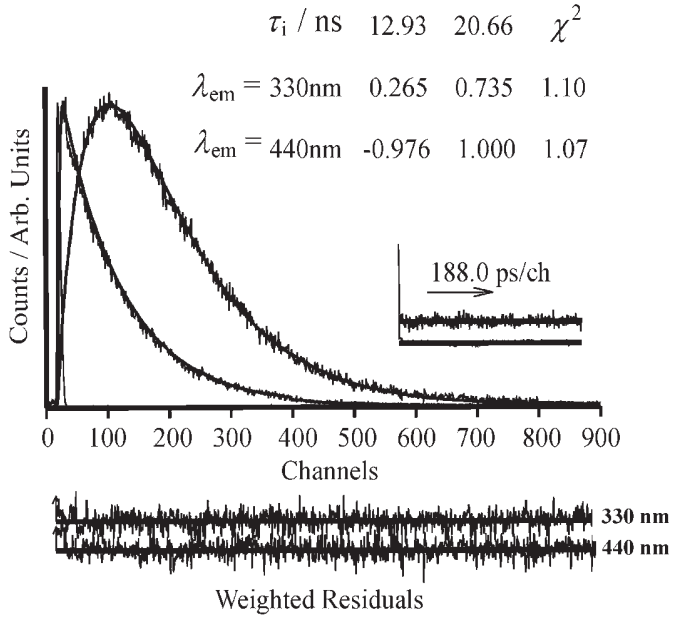

Figure 2. Fluorescence decays for Py/FN in isopropyl ether at $-10^{\circ} \mathrm{C}$ and fits obtained from the global analysis of the decays. The inset shows the decay times $\left(\tau_{\mathrm{i}} / \mathrm{ns}\right)$, the pre-exponential factors $\left(a_{i}\right)$, and the chi-squared values $\left(\chi^{2}\right)$. Also shown are the auto-correlation functions (A.C.) and the weighted residuals for a better judgment of the quality of the fits.

The monomer fluorescence-decay profiles are expected to become biexponential at temperatures at which the thermal CRIP dissociation reaction $\left(k_{\mathrm{d}}\right)$ cannot be neglected when compared with the intrinsic CRIP decay constant $\left(k_{\mathrm{E}}\right)$. The decrease in temperature leads, in the monomer emission region, to monoexponential decays, since $k_{\mathrm{E}} \gg k_{\mathrm{d}}$. In our kinetic analyses $k_{\mathrm{d}}$ is always present but the values obtained $\left(\approx 10^{4} \mathrm{~s}^{-1}\right)$ are always much lower than those of $k_{\mathrm{E}}\left(\approx 10^{8} \mathrm{~s}^{-1}\right)$ and of the fluorophore quenching rate constant, $k_{\mathrm{q}}=k_{\mathrm{a}} /[\mathrm{FN}]\left(\approx 10^{10} \mathrm{~s}^{-1} \mathrm{M}^{-1}\right.$, see Supporting Information). Figure 3 and Table 1 collect the relevant kinetic data. The ratio of the amplitudes $a_{12} / a_{11}$ also decreases with temperature, leading to zero values $\left(a_{11}=0\right.$ and $\mathrm{a}_{12}=1$; so that the ratio $\left.a_{12} / a_{11} \rightarrow \infty\right)$ and thus to monoexponential monomer decays at low temperatures.

The sum of the pre-exponential factors $\left(a_{22}\right.$ and $\left.a_{21}\right)$ of the decays collected at the CRIP emission region is practically equal to zero (see Figure 2). This shows that at the excitation wavelength, only the chromophore is being excited and, consequently, there is no ground-state association between pyrene (or naphthalene) and fumaronitrile. In isopropyl ether, all the CRIP are formed dynamically. In $n$-heptane, however, there are temperatures at which the decays do not show this behavior. Such cases are probably due to the strong contribution (overlap) of the monomer emission at the CRIP emission wavelength.

It is worth emphasizing that our kinetic model allows for reversibility, but it also shows that reversibility is negligible, which is in good agreement with the exothermicity calculated for the formation of the exciplexes $\left(\Delta G_{\mathrm{CS}}{ }^{0}<-10 \mathrm{kcal} \mathrm{mol}^{-1}\right)$.

In conclusion, the values of $\tau_{2}$ and $\tau_{\mathrm{E}}$ are practically indistinguishable within the experimental error and can therefore be entirely associated with the lifetime of the charge-separated state (see Table 1). As the fluorescence and triplet-formation quantum yields can be considered negligible, these values 

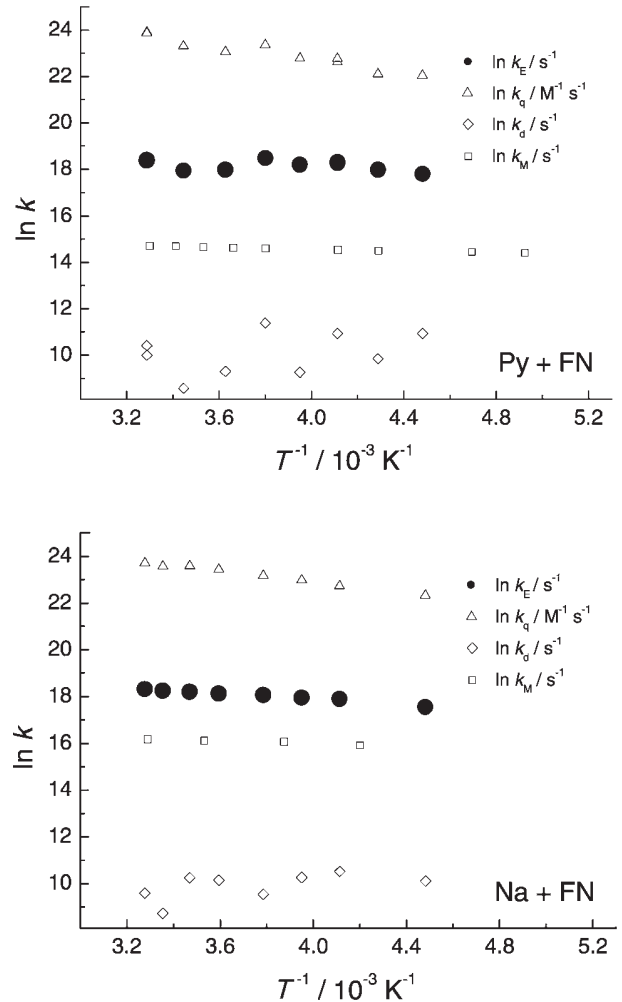

Figure 3. Temperature dependence (Arrhenius plots) of the monomer quenching, CRIP "dissociation", and decay rates in isopropyl ether. The reciprocals of the lifetimes of the monomers in the absence of a quencher are also shown.

quantitatively represent the charge recombination rate constants $\left(k_{\mathrm{CR}}=1 / \tau_{\mathrm{E}}\right)$.

\section{Discussion}

The free-energy dependence of the charge recombinations in isopropyl ether is similar to that observed in $n$-heptane and su- percritical $\mathrm{CO}_{2}{ }^{[15]}$ (Figure 4). The charge recombinations in Py/ $\mathrm{FN}$ and $\mathrm{Na} / \mathrm{FN} \mathrm{CRIP}$ are very exothermic and occur at the end of the inverted region, where the rates become very weakly dependent on $\Delta G^{0}{ }^{[23,24]}$ The observation of this new region was assigned to an increase in the reorganization energy $\lambda$ with an increase in $\left|\Delta G^{0}\right|$.

Figure 5 shows the crossing between the potential energy curves representing reactants and products when the increase in $\lambda$ exceeds the effect of $\Delta G^{0}$, becoming more negative, and the inverted region comes to an end.

The experimental data in Figure 4 clearly demonstrate that the charge recombinations in $n$-heptane and isopropyl ether at room temperature follow the same free-energy dependence. The $15 \mathrm{kcal} \mathrm{mol}^{-1}$ increase in the solvent reorganization energies, from $n$-heptane to isopropyl ether, calculated with Equation (3), has no influence on the kinetics of these reactions. This is an additional proof to the accumulating evidence that

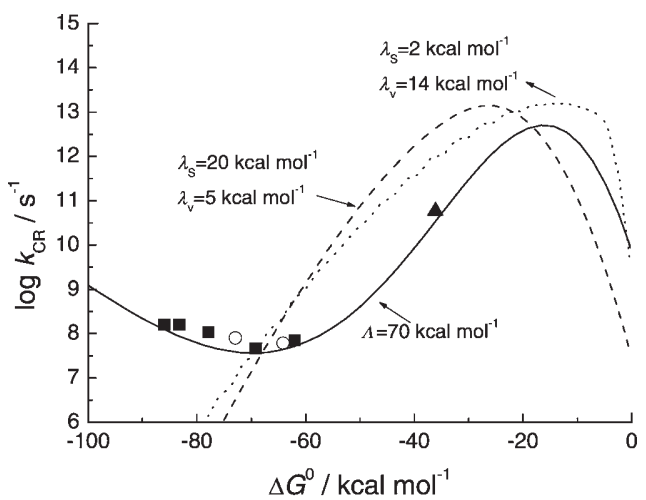

Figure 4. Free-energy dependence of charge-recombination rates in $n$-heptane $(\boldsymbol{\Xi})^{[15]}$ and cyclohexane $(\boldsymbol{\Delta})^{[36]}$ (at room temperature) and in isopropyl ether (O) [only rates measured at $17^{\circ} \mathrm{C}(\mathrm{Py} / \mathrm{FN})$ and $15^{\circ} \mathrm{C}(\mathrm{Na} / \mathrm{FN})$ are presented]. The golden rule calculations [dashed (-.--) and dotted $(. . .$.$) lines]$ employed $h v_{\mathrm{v}}=1500 \mathrm{~cm}^{-1}, V=300 \mathrm{~cm}^{-1}$, and the reorganization energies shown in the plot. The ISM calculations (-) employed $f=1.15 \times 10^{3} \mathrm{kcalmol}^{-1} \AA^{-2}, I_{\mathrm{r}}+I_{\mathrm{p}}=2.7 \AA, n^{\neq}=1.8, v=5 \times 10^{12} \mathrm{~s}^{-1}$, and the value of $\Lambda$ shown in the plot.

\begin{tabular}{|c|c|c|c|c|c|c|c|c|c|}
\hline$T\left[{ }^{\circ} \mathrm{C}\right]$ & $n_{\mathrm{D}}$ & $\varepsilon$ & $\begin{array}{l}\mathrm{Py} / \mathrm{FN} \\
\Delta G_{\mathrm{CR}}{ }^{0}\left[\mathrm{kcal} \mathrm{mol}^{-1}\right]\end{array}$ & $\tau_{2}[\mathrm{~ns}]$ & $k_{\mathrm{CR}}\left[10^{7} \mathrm{~s}^{-1}\right]$ & $\lambda\left[\mathrm{kcal} \mathrm{mol}^{-1}\right]$ & $\begin{array}{l}\mathrm{Na} / \mathrm{FN} \\
\Delta G_{\mathrm{CR}}^{0}\left[\mathrm{kcal} \mathrm{mol}^{-1}\right]\end{array}$ & $\tau_{2}[\mathrm{~ns}]$ & $k_{\mathrm{CR}}\left[10^{7} \mathrm{~s}^{-1}\right]$ \\
\hline 32 & 1.361 & 3.600 & -64.5 & & & 13.9 & -73.3 & 9.46 & 9.0 \\
\hline 31 & 1.361 & 3.639 & -64.5 & 7.95 & 9.7 & 14.1 & -73.3 & & \\
\hline 25 & 1.365 & 3.880 & -64.4 & & & 14.8 & -73.1 & 10.9 & 8.4 \\
\hline 17 & 1.371 & 4.227 & -64.1 & 14.2 & 6.1 & 15.7 & -72.9 & & \\
\hline 15 & 1.372 & 4.318 & -64.1 & & & 15.9 & -72.9 & 9.79 & 8.0 \\
\hline 5 & 1.379 & 4.806 & -63.9 & & & 16.9 & -72.7 & 10.9 & 7.3 \\
\hline 2.5 & 1.381 & 4.936 & -63.9 & 14.3 & 6.3 & 17.1 & -72.6 & & \\
\hline-10 & 1.390 & 5.642 & -63.7 & 7.88 & 10.5 & 18.1 & -72.4 & 12.9 & 7.0 \\
\hline-20 & 1.397 & 6.280 & -63.5 & 12.3 & 7.9 & 18.8 & -72.3 & 13.7 & 6.2 \\
\hline-30 & 1.404 & 6.989 & -63.4 & 11.4 & 8.8 & 19.3 & -72.1 & 14.6 & 5.9 \\
\hline-40 & 1.411 & 7.778 & -63.2 & 15.2 & 6.4 & 19.9 & -72.0 & & \\
\hline-50 & 1.418 & 8.656 & -63.1 & 17.0 & 5.3 & 20.3 & -71.9 & 20.8 & 4.2 \\
\hline
\end{tabular}

[a] The temperature dependence of the solvent parameters was taken from ref. [18], the oxidation potentials and the excited-state energies of the aromatic hydrocarbons from ref. [21], and the reduction potential of FN from ref. [22]; the reorganization energies of the Py/FN system were calculated with $r_{+}=$ 3.7 $\AA$ for pyrene and $r_{-}=2.8 \AA$ for fumaronitrile (obtained from the Connolly surfaces of these species, as in ref. [15]). 

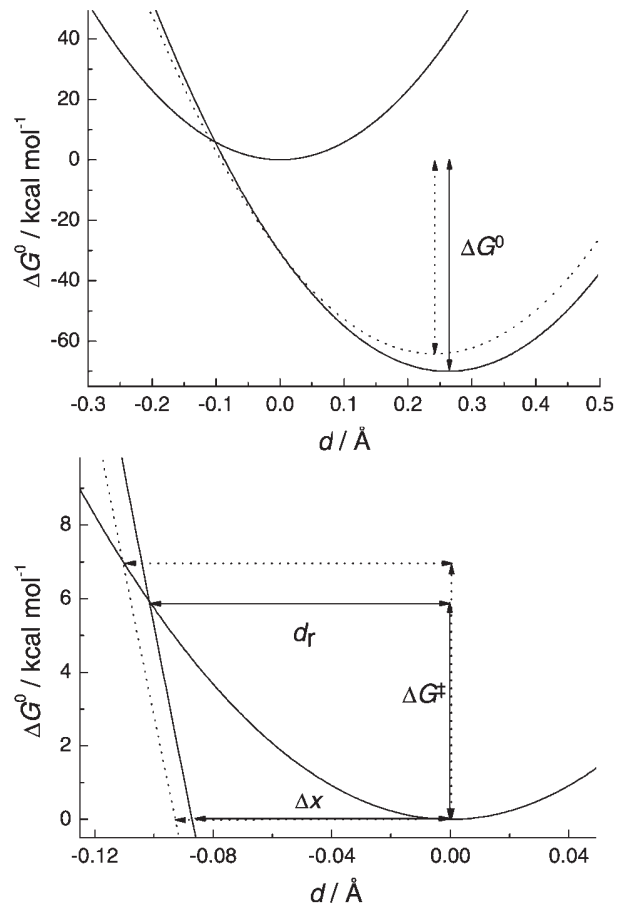

Figure 5. Top: Potential energy curves calculated with the parameters given in Figure 4. Py/FN (----), Na/FN (-). Bottom: Detail of the end of the inverted region, with the turnover from the typical increase of $\Delta G^{+}$with an increase of the exothermicity in the inverted region, to a decrease of $\Delta G^{\neq}$ with an increase of $\lambda$ with $\left|\Delta G^{0}\right|$.

the dielectric continuum model overemphasizes the role of the solvent in electron-transfer reactions. ${ }^{[6,15,24-28]}$ The data in this figure also show that we have reached a free-energy regime where small changes in $\Delta G^{0}$ have a negligible impact on $k_{\mathrm{CR}}$. Hence, we can study the temperature dependence of these charge recombinations without a significant perturbation by the $1.5 \mathrm{kcal} \mathrm{mol}^{-1}$ decrease in $\Delta G^{0}$ when the temperature is reduced by $80^{\circ} \mathrm{C}$, or by (small) unknown changes in the redox potentials with the temperature.

We recently showed ${ }^{[15]}$ that the free-energy relationship of Figure 4 can be reproduced by the golden rule if it is assumed that the reorganization energy increases with $\left|\Delta G^{0}\right|$. Unfortunately, the reorganization energy then becomes an adjustable parameter, which is further complicated by the fact that changes in $\lambda_{s}$ and $\lambda_{v}$ compensate for each other, as illustrated in Figure 4. The lifetimes of the Py/FN and $\mathrm{Na} / \mathrm{FN}$ CRIP are nearly independent of the temperature. The golden rule [Eqs. (1) and (2)] can reproduce the charge-recombination rates in these CRIPs using temperature-independent reorganization energies for each system; for example, a good fit to the Py/FN charge recombination can be obtained with $\lambda_{s}=20 \mathrm{kcal} \mathrm{mol}^{-1}$, $\lambda_{v}=5 \mathrm{kcalmol}^{-1}, h v_{v}=1500 \mathrm{~cm}^{-1}$, and $V=300 \mathrm{~cm}^{-1}$. This would be appropriate for $-50^{\circ} \mathrm{C}$, but according to Equation (3), $\lambda_{s}$ should decrease to $14 \mathrm{kcal} \mathrm{mol}^{-1}$ at $32^{\circ} \mathrm{C}$. With the temperature dependence of $\lambda_{s}$ given by Equation (3), the golden rule predicts an increase in the rates with a decrease in the temperature which is not observed (Figure 6). Temperature-independent rates can also be calculated with the golden rule using a large $\lambda_{v}$ and a small $\lambda_{s}$. This combination of param-

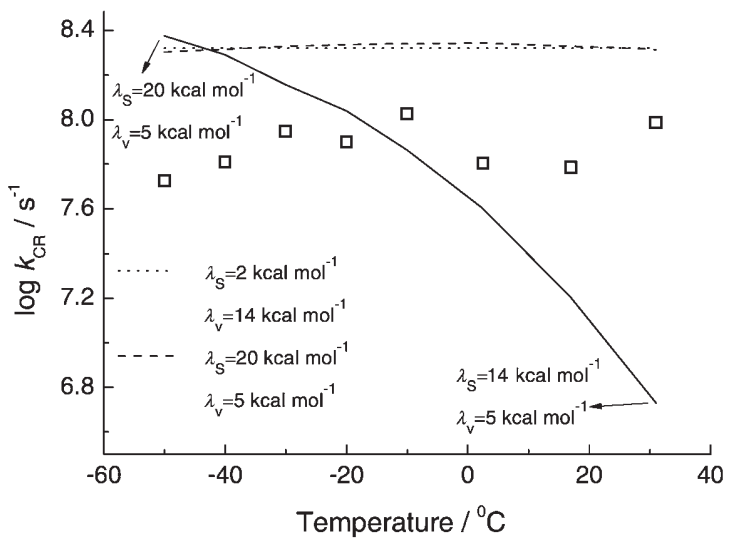

Figure 6. Temperature dependence of charge-recombination rates in the $\mathrm{Py} /$ FN CRIP in isopropyl ether ( $\square$ ). Dashed (--.--) and dotted (.....) lines: golden rule calculations with constant reorganization energies; solid line ( - ): golden rule calculations with solvent reorganization energies varying according to the dielectric continuum approximation.

eters is more consistent with the kinetic data measured on different media (polar and nonpolar organic solvents, porous glasses, supercritical fluids), because the calculations become less sensitive to the properties of that media. This provides further evidence that the dielectric continuum model overemphasizes the role of the solvent in electron-transfer reactions.

Jortner and Ulstrup have shown ${ }^{[29]}$ that for isoenergetic processes, the Franck-Condon factor that represents the overlap between the ground vibrational wavefunction of the initial state and the vibrational wavefunction of the final electronic state takes the form [Eq. (11)]:

$J_{0}=C \exp \left[-\frac{\sqrt{2 \mu \Delta E^{\ddagger} \Delta x}}{h} \tanh \left(h v / 4 k_{\mathrm{B}} T\right)\right]$

where we neglected the dependence of the pre-exponential factor $C$. In the expression above, $\mu$ is the reduced mass of the tunneling particle, $\Delta E^{+}$the reaction barrier, and $\Delta x$ its width measured isoenergetically from the reactant to the product curve, as illustrated in Figure 5. For $h v_{v}=1500 \mathrm{~cm}^{-1}$ and $T=$ $223 \mathrm{~K}$, the hyperbolic function has the value 0.984 , and it is a good approximation to write the exponential term as the Wentzel-Kramers-Brillouin (WKB) approximation for tunneling through the barrier formed by the intersecting parabolas [Eq. (12)]: ${ }^{[30]}$

$k_{\mathrm{tun}}=v \exp \left[-\frac{\sqrt{2 \mu \Delta E^{\ddagger}} \Delta x}{\hbar}\right]$

where $v$ is the reaction frequency. The isomorphism between the equations expressing the Franck-Condon factors and the WKB approximation for tunneling [Eqs. (11) and (12)] is a heuristic validation of the latter. We calculated the barrier width and height from the experimental reaction energy $\left(\Delta G^{0}\right)$ and the separation between the reactant and product minima along the reaction coordinate $(d)$. This last parameter can be 
estimated using the intersecting-state model (ISM). ${ }^{[2]}$ The tunneling rates were then calculated with Equation (12).

According to the ISM, the sum of the reactant and product bond extension along the reaction coordinate is given by Equation (13):

$d=\frac{a^{\prime}}{2 n^{\mp}} \ln \left\{\frac{1+\exp \left(\sqrt{2 n^{\mp}} \Delta G^{0} / \Lambda\right)}{1-\left[1+\exp \left(\sqrt{2 n^{\mp}} \Delta G^{0} / \Lambda\right)\right]^{-1}}\right\}\left(I_{r}+I_{p}\right)$

where $a^{\prime}$ is a constant $\left(a^{\prime}=0.156\right), n^{\neq}$is the transition-state bond order, $I_{\mathrm{r}}\left(I_{\mathrm{p}}\right)$ represents the bond lengths of the reactants (products) that participate in the reaction coordinate, and the parameter $\Lambda$ is associated with the dissipation of the reaction energy $\Delta G^{0}$. In the present calculations we employ the same parameters previously employed for these systems: $n^{\neq}=1.8$, $I_{\mathrm{r}}+I_{\mathrm{p}}=2.7 \AA$, and $\Lambda=70 \mathrm{kcal} \mathrm{mol}^{-1} \cdot{ }^{[15]}$ The free-energy relationship obtained with these parameters and the thermal activation [Eq. (14)]:

$\Delta G^{\neq}=\frac{1}{2} f_{r} d_{r}^{2}$

where $f=1.15 \times 10^{3} \mathrm{kcal} \mathrm{mol}^{-1} \AA^{-2}$ is the force constant associated with the frequency $1500 \mathrm{~cm}^{-1}$, the reduced mass of a CC oscillator, and a reaction frequency of $v=5 \times 10^{12} \mathrm{~s}^{-1}$, is shown in Figure 4. The tunneling rate constants can be calculated with Equation (12), provided that the reduced mass is known.

The effective reduced mass has contributions from the active modes of the aromatic hydrocarbon and of fumaronitrile. Considering that both must tunnel through the same barrier, that is, $\Delta E^{\ddagger}$ and $\Delta x$ are the same for both reactants, then [Eq. (15)]:

$\sqrt{\mu}=\sqrt{\mu_{\mathrm{Ar}}}+\sqrt{\mu \mathrm{F}}$

The reaction coordinate was built from the vibrational frequencies, reduced masses, and bond lengths of the CC oscillators. For consistency, $\mu_{\mathrm{Ar}}$ must also be given by these oscillators, for which $\mu_{\mathrm{cC}}=6 \mathrm{amu}$. In naphthalene there are five such oscillators, hence, $\mu_{\mathrm{Na}}=30 \mathrm{amu}$, in pyrene there are eight so that $\mu_{\mathrm{Py}}=48 \mathrm{amu}$. This procedure to obtain the effective oscillator representing each reactant is mathematically identical to that employed by McCoy and Ross to express the total bondlength-displacement coordinate of polycyclic aromatic hydrocarbons in terms of the individual bond-length displacements of each oscillator [Eq. (16)]: ${ }^{[31]}$

$\Delta X=\left(\sum_{i} \Delta x_{i}^{2}\right)^{1 / 2}$

Identically, in fumaronitrile we have two $\mathrm{CN}$ oscillators and one CC oscillator; thus, $\mu_{\mathrm{FN}}=18.9 \mathrm{amu}$. The reduced masses in Equation (15), together with $v=5 \times 10^{12} \mathrm{~s}^{-1}$ in Equation (12), give the tunneling rates illustrated in Figure 7.

The tunneling rates are in very good agreement with the low-temperature rates. Thermal activation competes with tun-
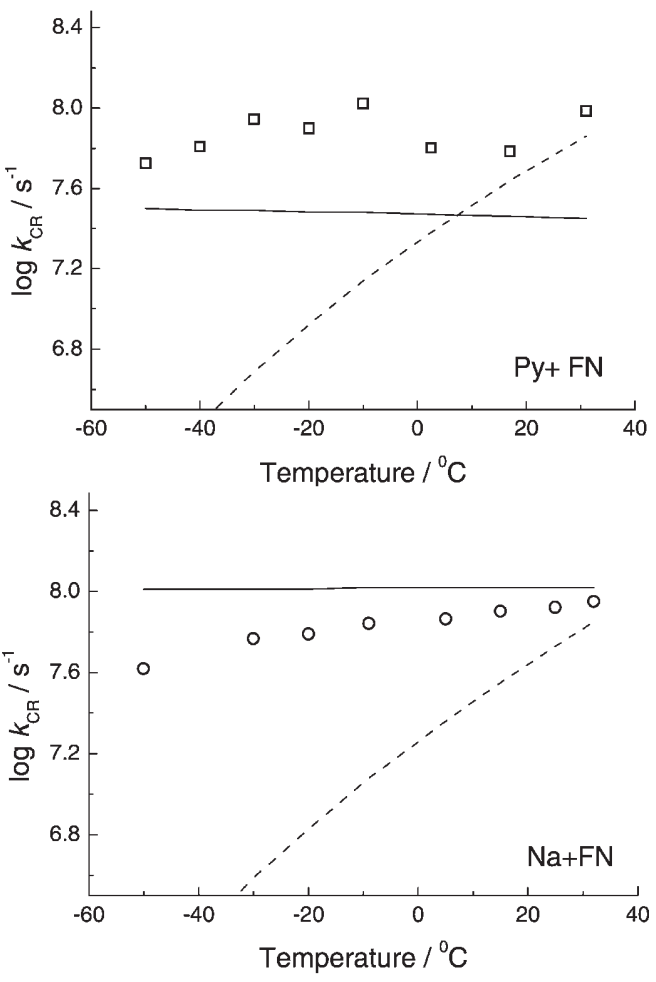

Figure 7. Temperature dependence of charge-recombination rates in: Py/FN (口) (top) and $\mathrm{Na} / \mathrm{FN}(\mathrm{O})$ (bottom) CRIPs. Dashed lines (-----): ISM calculations for thermal activation using the parameters in Figure 4; solid lines (-): ISM calculations for nuclear tunneling using the parameters in Figure 4 and $\mu_{\mathrm{Py} / \mathrm{FN}}=127 \mathrm{amu}$ or $\mu_{\mathrm{Na} / \mathrm{FN}}=96.5 \mathrm{amu}$.

neling at higher temperatures. The two systems differ slightly because the effective reduced mass calculated for naphthalene is smaller (more tunneling) than for pyrene. Unfortunately, this difference is within the order-of-magnitude accuracy of the calculations and cannot be completely tested with these systems.

The temperature dependence of the CRIP charge-recombination rates reported here is very similar to that of the intramolecular charge recombinations measured by Verhoeven and co-workers in hydrocarbon solvents for similar redox-active species. However, it differs from that reported by Miyasaka and co-workers, ${ }^{[6]}$ who show the presence of an activated regime at higher temperatures. The origin of this activated regime cannot be assigned to the decrease or freezing of the intermolecular vibration of the two radical ions, because this vibration is also present in our system (and we observe no activated regime). It is tempting to assign it to the heavier masses involved in the work by Miyasaka et al. (tetracyanobenzene versus fumaronitrile), but our calculations, with the reduced mass given by Equation (15), underestimate the ratios $k_{\mathrm{CR}^{-}}$ $(293 \mathrm{~K}) / k_{\mathrm{CR}}(77 \mathrm{~K})$ measured by these authors. It is perhaps unwarranted to expect that this simple model could account for such fine details of the temperature dependence of electrontransfer reactions, but it is also fair to refer the success of this tunneling model in reproducing the temperature dependence of the photosynthetic bacteria reaction centre. ${ }^{[32]}$

Following Wynne et al. ${ }^{[33]}$ we also assigned an important role to the intermolecular vibration of the two radical ions. It is 
strongly coupled to the reaction coordinate and it is an important accepting mode for the disposal of the reaction energy $\Delta G^{0}$. However, the weak temperature dependence of charge recombinations in CRIPs is not surprising because the disposal of $60 \mathrm{kcal} \mathrm{mol}^{-1}$ in $10 \mathrm{~ns}$ produces a very large transient local heating that contributes more to the population of the $165 \mathrm{~cm}^{-1}$ donor/acceptor stretching than the temperatures experimentally accessible.

\section{Conclusions}

The CRIPs formed between aromatic hydrocarbon cations and the fumaronitrile anion are weakly fluorescent and their decays are controlled by charge recombination. The charge recombinations measured herein have exothermicities between -60 and $-75 \mathrm{kcal} \mathrm{mol}^{-1}$ and fall within a regime in which the rates are weakly dependent on the free energies of reaction. The charge-recombination rates measured in isopropyl ether and $n$-heptane are very similar to each other, showing that the polarity of the solvent has a negligible influence on them. Additionally, these rates remain approximately constant in the range of temperatures studied in this work, which is also in conflict with the solvent-reorganization energies estimated with the dielectric continuum model. The temperature independence of these rates is reasonably well reproduced by a simple tunneling model, which emphasizes the nature of the mechanism involved in these reactions.

\section{Experimental Section}

Absorption and fluorescence spectra were recorded on Shimadzu UV-2100 and Horiba-Jobin-Ivon SPEX Fluorog 3-22 spectrometers, respectively. The fluorescence spectra were corrected for the wavelength response of the system. The low optical density of the samples used prevents self-absorption or inner-filter effects. Fluorescence decays were measured using a home-built time-correlated single-photon-counting (TCSPC) apparatus as described elsewhere, ${ }^{[34]}$ except that a Horiba-JI-IBH NanoLED, $\left(\lambda_{\text {exc }}=339 \mathrm{~nm}\right.$ or $281 \mathrm{~nm}$ ) was used as the excitation source. The fluorescence decays were analyzed using the "modulating functions" method of Striker et al. ${ }^{[35]}$ Temperature control was achieved using a homebuilt system based on cooled nitrogen and electric heating, which is automatically controlled by the difference between the input temperature value and the real temperature of the sample determined with a PT100 thermometer. Sample solutions were gentle bubbled with argon for 45 min prior to every experiment.

Naphthalene (Aldrich, 99.8\%) and pyrene (Aldrich, +99\%) were zone-refined. Fumaronitrile (Aldrich, 98\%) was vacuum-sublimed. Isopropyl ether (Merck, 99\%) was dried over $\mathrm{CaSO}_{4}$, distilled over sodium pentoxide, and passed thorough an alumina column just before use. $n$-heptane (Aldrich, spectroscopic grade) was directly used after convenient drying over sodium.

\section{Acknowledgments}

We thank Fundação para a Ciência e Tecnologia (Portugal) and FEDER (European Union) for financial support; project no. POCTI/
QUI/47267/2002. C.S. acknowledges FCT (Portugal) for grant SFRH/BPD/13297/2003.

Keywords: charge recombination - electron transfer · ion pairs $\cdot$ temperature dependence $\cdot$ tunneling

[1] R. A. Marcus, Faraday Discuss. Chem. Soc. 1960, 29, 21.

[2] N. Liang, J. R. Miller, G. L. Closs, J. Am. Chem. Soc. 1989, 111, 8740.

[3] N. Liang, J. R. Miller, G. L. Closs, J. Am. Chem. Soc. 1990, 112, 5353.

[4] J. Kroon, H. Oevering, J. W. Verhoeven, J. M. Warman, A. M. Oliver, M. N. Paddon-Row, J. Phys. Chem. 1993, 97, 5065.

[5] M. N. Paddon-Row, A. M. Oliver, J. M. Warman, K. J. Smit, M. P. Haas, H. Oevering, J. W. Verhoeven, J. Phys. Chem. 1988, 92, 6958.

[6] H. Miyasaka, S. Kotani, A. Itaya, G. Schweitzer, F. C. De Schryver, N. Mataga, J. Phys. Chem. B 1997, 101, 7978.

[7] M. Van der Auweraer, A. Gilbert, F. C. De Schryver, J. Am. Chem. Soc. 1979, 102, 4007.

[8] J. P. Palmans, A. M. Swinnen, G. Desie, M. Van der Auweraer, J. Vanderdriessche, F. C. De Schryver, J. Photochem. 1985, 28, 419.

[9] R. A. Marcus, J. Chem. Phys. 1956, 24, 966.

[10] R. P. Van Duyne, S. F. Fischer, Chem. Phys. 1974, 5, 183.

[11] N. R. Kestner, J. Logan, J. Jortner, J. Phys. Chem. 1974, 78, 2148.

[12] S. Efrima, M. Bixon, Chem. Phys. Lett. 1974, 25, 34.

[13] R. A. Marcus, N. Sutin, Biochim. Biophys. Acta 1985, 811, 265.

[14] A. Weller, Z. Physik. Chem. N. F. 1982, 133, 93.

[15] C. Serpa, P. J. S. Gomes, L. G. Arnaut, S. J. Formosinho, J. Pina, J. Seixas de Melo, Chem. Eur. J. 2006, 12, 5014.

[16] P. Van Haver, N. Helsen, S. Depaemelaere, M. Van der Auweraer, F. C. De Schryver, J. Am. Chem. Soc. 1991, 6849.

[17] I. R. Gould, R. H. Young, L. J. Mueller, A. C. Albrecht, S. Farid, J. Am. Chem. Soc. 1994, 116, 8188.

[18] Y. Marcus, The Properties of Solvents, Vol. 4, J. Wiley \& Sons, Chichester, 1998.

[19] F. Bouwer in Conformational Analysis of Molecules in Excited States (Ed.: J. Waluk), Wiley-VCH, New York, 2000.

[20] J. B. Birks, Photophysics of aromatic molecules, Wiley, London, 1970

[21] S. L. Murov, I. Carmichael, G. L. Hug, Handbook of Photochemistry, 2nd ed., Marcel Dekker, Inc., New York, 1993.

[22] J. P. Petrovich, M. M. Baizer, M. R. Ort, J. Electrochem. Soc. 1969, 116, 743.

[23] L. G. Arnaut, S. J. Formosinho, J. Mol. Struct. (Theochem) 1991, 233, 209.

[24] S. J. Formosinho, L. G. Arnaut, R. Fausto, Prog. Reaction Kinetics 1998, 23, 1.

[25] C. Serpa, L. G. Arnaut, J. Phys. Chem. A 2000, 104, 11075.

[26] S. F. Nelsen, A. Konradsson, T. L. Jentzsch, J. J. O'Konek, J. R. Pladziewicz, J. Chem. Soc., Perkin Trans. 2 2001, 1552.

[27] M. Dossot, P. Jacques, Helv. Chim. Acta 2001, 84, 3446.

[28] K.-X. Fu, X.-X. Li, Q. Zhu, Z. Gong, S.-Z. Lu, Z.-M. Bao, J. Mol. Struct. (Theochem) 2005, 715, 157

[29] J. Jortner, J. Ulstrup, Chem. Phys. Lett. 1979, 63, 236.

[30] S. J. Formosinho, J. Chem. Soc., Faraday Trans. 2 1974, 70, 605.

[31] E. F. McCoy, I. G. Ross, Aust. J. Chem. 1962, 15, 573.

[32] L. G. Arnaut, S. J. Formosinho, J. Photochem. Photobiol. A: Chem. 1997, $111,111$.

[33] K. Wynne, G. D. Reid, R. M. Hochstrasser, J. Chem. Phys. 1996, 105, 2287.

[34] J. Seixas de Melo, P. F. Fernandes, J. Mol. Struct. 2001, 565, 69.

[35] G. Striker, V. Subramaniam, C. A. M. Seidel, A. Volkmer, J. Phys. Chem. B 1999, 103, 8612.

[36] K. Wynne, C. Galli, R. M. Hochstrasser, J. Chem. Phys. 1994, 1000, 4797.

Received: July 31, 2006

Published online on October 30, 2006 